\title{
Conductivity scaling and the effects of symmetry-breaking terms in bilayer graphene Hamiltonian
}

\author{
Dominik Suszalski, ${ }^{1}$ Grzegorz Rut, ${ }^{1,2}$ and Adam Rycerz ${ }^{1}$ \\ ${ }^{1}$ Marian Smoluchowski Institute of Physics, Jagiellonian University, Łojasiewicza 11, PL-30348 Kraków, Poland \\ ${ }^{2}$ CRIF sp. z o.o., Lublańska 38, PL-31476 Kraków, Poland
}

(Received 9 December 2019; accepted 11 March 2020; published 30 March 2020)

\begin{abstract}
We study the ballistic conductivity of bilayer graphene in the presence of symmetry-breaking terms in an effective Hamiltonian for low-energy excitations, such as the trigonal-warping term $\left(\gamma_{3}\right)$, the electron-hole symmetry-breaking interlayer hopping $\left(\gamma_{4}\right)$, and the staggered potential $\left(\delta_{A B}\right)$. Earlier, it was shown that for $\gamma_{3} \neq 0$, in the absence of remaining symmetry-breaking terms (i.e., $\gamma_{4}=\delta_{A B}=0$ ), the conductivity $(\sigma)$ approaches the value of $3 \sigma_{0}$ for the system size $L \rightarrow \infty$ [with $\sigma_{0}=8 e^{2} /(\pi h)$ being the result in the absence of trigonal warping, $\gamma_{3}=0$ ]. We demonstrate that $\gamma_{4} \neq 0$ leads to the divergent conductivity $(\sigma \rightarrow \infty)$ if $\gamma_{3} \neq 0$, or to the vanishing conductivity $(\sigma \rightarrow 0)$ if $\gamma_{3}=0$. For realistic values of the tight-binding model parameters, $\gamma_{3}=0.3 \mathrm{eV}, \gamma_{4}=0.15 \mathrm{eV}$ (and $\delta_{A B}=0$ ), the conductivity values are in the range $\sigma / \sigma_{0} \approx 4-5$ for $100 \mathrm{~nm}<L<1 \mu \mathrm{m}$, in agreement with existing experimental results. The staggered potential $\left(\delta_{A B} \neq 0\right)$ suppresses zero-temperature transport, leading to $\sigma \rightarrow 0$ for $L \rightarrow \infty$. Although $\sigma=\sigma(L)$ is no longer universal, the Fano factor approaches the pseudodiffusive value $(F \rightarrow 1 / 3$ for $L \rightarrow \infty)$ in any case with nonvanishing $\sigma$ (otherwise, $F \rightarrow 1$ ), signaling the transport is ruled by evanescent waves. Temperature effects are briefly discussed in terms of a phenomenological model for staggered potential $\delta_{A B}=\delta_{A B}(T)$ showing that, for $0<T \leqslant T_{c} \approx 12 \mathrm{~K}$ and $\delta_{A B}(0)=1.5 \mathrm{meV}, \sigma(L)$ is noticeably affected by $T$ for $L \gtrsim 100 \mathrm{~nm}$.
\end{abstract}

DOI: 10.1103/PhysRevB.101.125425

\section{INTRODUCTION}

The universal conductivity of monolayer graphene (MLG), $\sigma_{\mathrm{MLG}}=4 e^{2} /(\pi h)$ (with the elementary charge $e$ and the Planck constant $h$ ), accompanied by the pseudodiffusive shot noise (quantified by the Fano factor $F=1 / 3$ ), is one of the most recognizable landmarks of the Dirac nature of electrons that dwell in this material [1-5]. These unique characteristics are linked to the dominant role of transport via evanescent waves in graphene near the charge-neutrality point [6]. What is more, the effective Hamiltonian for low-energy excitations,

$$
H_{\mathrm{MLG}}=v_{F}\left(p_{x} \sigma_{x}+p_{y} \sigma_{y}\right),
$$

where $v_{F}=\sqrt{3} t_{0} a /(2 \hbar) \approx 10^{6} \mathrm{~m} / \mathrm{s}$ is the energyindependent Fermi velocity (with $t_{0} \approx 3 \mathrm{eV}$ the nearestneighbor hopping integral and $a=0.246 \mathrm{~nm}$ the lattice spacing), $p_{j}=-i \hbar \partial_{j}$ are in-plane momentum operators, and $\sigma_{j}$ are the Pauli matrices acting on the sublattice degree of freedom (with $j=x, y$ ), possesses several symmetries which are crucial for the simplicity of transport properties. (We further note that the absence of valley-coupling factors is supposed throughout the paper, and the discussion is limited to the $K$ valley.) These include the rotational invariance (RI), the electron-hole symmetry (EHS), and the sublattice equivalence (SE), which is embedded in the so-called

Published by the American Physical Society under the terms of the Creative Commons Attribution 4.0 International license. Further distribution of this work must maintain attribution to the author(s) and the published article's title, journal citation, and DOI. symplectic symmetry (or time-reversal symmetry in a single valley) $[7,8]$.

In bilayer graphene (BLG) the situation is more complex due to the couplings between the layers [9-13]. Historically, the effective Hamiltonians for BLG were constructed by taking only the leading tight-binding parameters of the Slonczewski-Weiss-McClure model [14,15], which are indicated in Fig. 1(a).

Even in the simplest possible approach $[9,10]$, including the nearest-neighbor interlayer hopping $\gamma_{0}$ (being numerically different than $t_{0}$ ) and the direct interlayer hopping $\gamma_{1}$, SE is already eliminated due to the inequivalence of sites connected by $\gamma_{1}$ (dimer sites) and the remaining ones (nondimer sites), giving an opportunity to open the band gap by perpendicular electric field introducing the layer inequivalence [16]. (The second-nearest-neighbor interlayer hopping, formally breaking EHS, is usually omitted as - in the low-energy limit-it only shifts the charge-neutrality point by a constant value; see Ref. [17].) Quite surprisingly, the approach of Refs. [9,10] leads to the conductivity $\sigma_{0}=2 \sigma_{\mathrm{MLG}}=8 e^{2} /(\pi h)$ and $F=$ $1 / 3$ (in the absence of a gap), as one could expect for two decoupled layers. The results are also size independent, provided that $W \gg L \gg l_{\perp}$, with the sample width $W$, the length $L$ marked in Fig. $1(\mathrm{~b})$, and $l_{\perp}=\sqrt{3} a \gamma_{0} /\left(2 \gamma_{1}\right) \approx 1.77 \mathrm{~nm}$ being a new length scale due the coupling between the layers.

Next, skew-interlayer hopping (or the trigonal-warping term) $\gamma_{3}$ [18] breaks RI, leading to the appearance of three additional Dirac cones at each valley [16]. The effect of $\gamma_{3}$ on quantum transport is also significant [11-13]; namely, the conductivity $\sigma(L)$ is no longer universal but length dependent, approaching the value of $3 \sigma_{0}$ for large $L$ [19]. In contrast, 
(a)

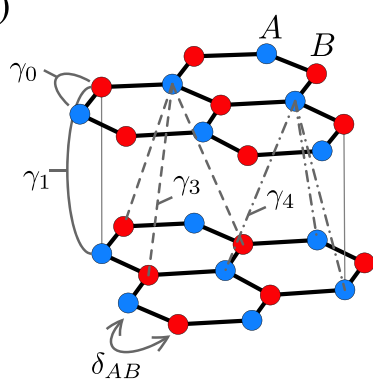

(b)

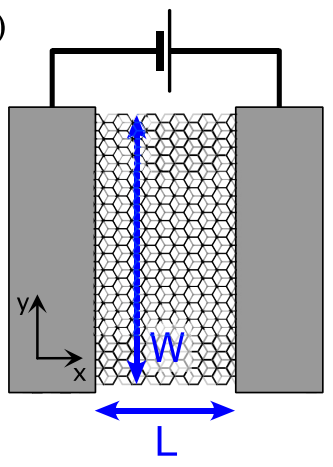

symmetry breaking. The concluding remarks are given in Sec. IV.

The numerical results presented in the main text are supplemented with the explicit mode-matching analysis for the special cases of $\gamma_{3} \neq 0, \gamma_{4}=\delta_{A B}=0$ (Appendix A) and $\gamma_{3}=$ $\delta_{A B}=0, \gamma_{4} \neq 0$ (Appendix B).

\section{THE MODEL}

We start from the minimal version of the four-band Hamiltonian [16], in which all the symmetry breakings mentioned in Sec. I are quantified by independent parameters:

$$
H_{\mathrm{BLG}}=\left(\begin{array}{cccc}
\delta_{A B} / 2 & v_{0} \pi & \gamma_{1} & -v_{4} \pi^{\dagger} \\
v_{0} \pi^{\dagger} & -\delta_{A B} / 2 & -v_{4} \pi^{\dagger} & v_{3} \pi \\
\gamma_{1} & -v_{4} \pi & -\delta_{A B} / 2 & v_{0} \pi^{\dagger} \\
-v_{4} \pi & v_{3} \pi^{\dagger} & v_{0} \pi & \delta_{A B} / 2
\end{array}\right),
$$

where $\pi=e^{-i \theta}\left(p_{x}+i p_{y}\right), \pi^{\dagger}=e^{i \theta}\left(p_{x}-i p_{y}\right)$, with the angle $\theta$ (between an armchair direction and the $x$ axis) defining the crystallographic orientation of the sample, $v_{0}=\sqrt{3} a \gamma_{0} /(2 \hbar)$, $v_{3}=v_{0} \gamma_{3} / \gamma_{0}$, and $v_{4}=v_{0} \gamma_{4} / \gamma_{0}$. In the forthcoming numerical discussion, we set $\theta=\pi / 4, \gamma_{0}=3.16 \mathrm{eV}$, and $\gamma_{1}=$ $0.381 \mathrm{eV}$ [24]; for each of the remaining parameters the cases of zero and nonzero value are studied independently to demonstrate the impact of a particular symmetry breaking on ballistic transport. Namely, we took $\gamma_{3}=0$ or $0.3 \mathrm{eV}, \gamma_{4}=0$ or $0.15 \mathrm{eV}$, and $\delta_{A B}=0$ or $1.5 \mathrm{meV}$.

Our specific choice of the staggered potential $\delta_{A B}$ in the Hamiltonian $H_{\mathrm{BLG}}$ (2) follows from the demand that it opens a band gap without breaking EHS, which is solely controlled by $\gamma_{4}$. (In the parametrization of Ref. [16] the energy difference between dimer and nondimer sites $V$ also breaks EHS; here we set $V=0$ ). Physically, $\delta_{A B}$ represents the irreducible part of a gap (i.e., one that cannot be closed by external electric fields) and can be attributed to charge or spin order which may appear in the BLG ground state when electron-electron repulsive interactions are taken into account $[25,26]$.

In Fig. 2 we present the low-energy band structure following from the Hamiltonian $H_{\mathrm{BLG}}(2)$ by displaying the cross sections, for $p_{y}=\hbar k_{y}=0$, of dispersion relations for eight different combinations of symmetry-breaking parameters $\gamma_{3}, \gamma_{4}$, and $\delta_{A B}$. An apparent feature visible in Fig. 2(a) is the energy shift of a secondary Dirac cone (same for all three secondary cones) due to EHS breaking for $\gamma_{4} \neq 0$ [see Fig. 2(c) for a comparison], making it impossible (for $\gamma_{3} \neq 0$ and $\left.\delta_{A B}=0\right)$ to achieve the exact zero-doping case, in which the transport is fully carried by evanescent waves. In contrast, for $\gamma_{3}=0$ [see Figs. 2(e) and 2(f)], the effects of $\gamma_{4}$ are marginal (apart from clear electron-hole asymmetries visible for $\gamma_{4} \neq 0$ ), and the zero-doping case can be achieved for both $\gamma_{4}=0$ or $\gamma_{4} \neq 0$. For $\delta_{A B} \neq 0$, we have an indirect band gap for $\gamma_{4} \neq 0$ and $\gamma_{4} \neq 0$ [see Fig. 2(b)], or direct band gaps in the remaining cases, allowing one to obtain the zero doping by adjusting the Fermi level to the gap.

The consequences of these features for BLG transport properties are discussed next. 

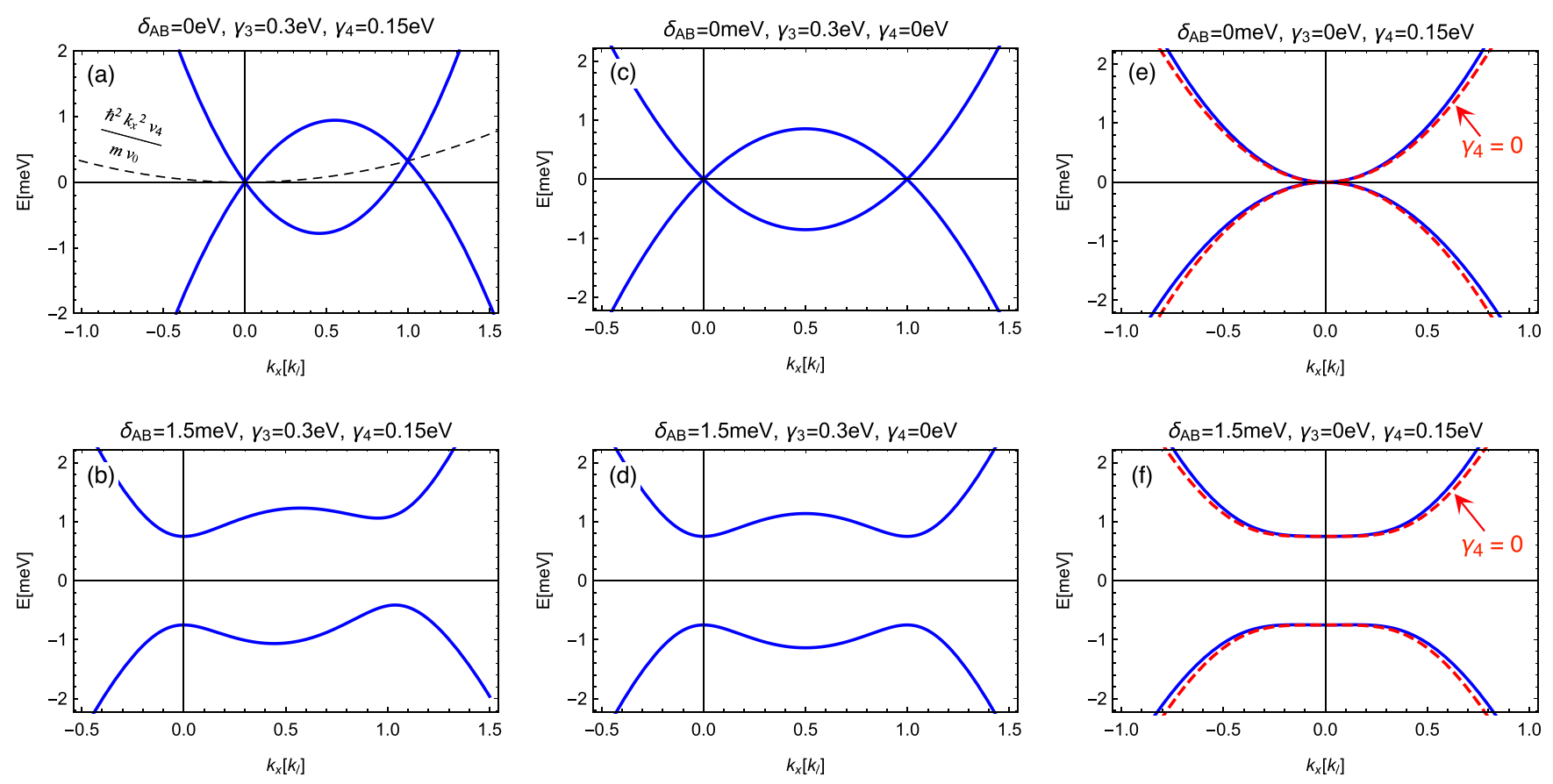

FIG. 2. (a)-(f) Band energies for the Hamiltonian $H_{\text {BLG }}$ given by Eq. (2) with $\theta=0$. Solid lines correspond to the parameters $\left(\delta_{A B}, \gamma_{3}, \gamma_{4}\right)$ specified in each panel. The dashed black line in (a) marks the parabolic correction due to the electron-hole symmetry breaking interlayer hopping $\gamma_{4}$ (see the explicit formula with $m=\gamma_{1} / 2 v_{0}^{2}$ ). The dashed red lines in (e) and (f) depict the reference band structure for $\gamma_{3}=\gamma_{4}=0$. The wavenumber $k_{x}=p_{x} / \hbar$ is specified in units of $k_{l}=\frac{2}{3} \sqrt{3} \gamma_{1} \gamma_{3} /\left(a \gamma_{0}^{2}\right) \approx 0.05 \mathrm{~nm}^{-1}$, being the $k_{x}$ position of a secondary Dirac cone calculated for the parameters as listed in (c). Each panel displays the cross section taken at $k_{y}=0$.

\section{RESULTS AND DISCUSSION}

\section{A. Zero-temperature charge transport}

We employ the Landauer-Büttiker expressions for zerotemperature conductivity and the Fano factor in the linearresponse regime [27], namely,

$$
\begin{gathered}
\sigma(T \rightarrow 0)=\frac{g_{0} L}{W} \operatorname{Tr}\left(\mathbf{t} \mathbf{t}^{\dagger}\right), \\
F=\frac{\operatorname{Tr}\left[\mathbf{t t}^{\dagger}\left(\mathbf{1}-\mathbf{t t}^{\dagger}\right)\right]}{\operatorname{Tr}\left(\mathbf{t} \mathbf{t}^{\dagger}\right)}
\end{gathered}
$$

with the conductance quantum $g_{0}=4 e^{2} / h$ accounting for spin and valley degeneracies. In order to determine the transmission matrix at a given Fermi energy, $\mathbf{t}=\mathbf{t}(E)$, for a rectangular sample attached to the two heavily doped regions, we employ the computational scheme similar to that presented in Ref. [13], with a numerical stabilization introduced in Ref. [28]. In brief, at finite-precision arithmetics, the modematching equations may become ill defined for sufficiently large $L$, as they contain both exponentially growing and exponentially decaying coefficients. This difficulty is overcome by dividing the sample area into $N_{\text {div }}$ consecutive, equally long parts and matching the wave functions for all (i.e., $N_{\text {div }}+1$ ) interfaces. Typically, using the double-precision arithmetic, we put $N_{\text {div }}=\left\lfloor L /\left(40 l_{\perp}\right)\right\rfloor+1$, with $\lfloor x\rfloor$ denoting the floor of $x$.

Our numerical results for $E=0$ are presented in Figs. 3 and 4. As the debate on the ground-state nature of BLG is currently ongoing [26] and the existing experimental results are far from being consistent [20-23,29,30], we examine eight possible scenarios by setting different values of the parameters $\left(\delta_{A B}, \gamma_{3}, \gamma_{4}\right)$ in the low-energy Hamiltonian $H_{\mathrm{BLG}}(2)$, corresponding to the dispersion relations presented in Sec. II.

The behavior of transport properties is relatively simple for $\delta_{A B}=1.5 \mathrm{meV}$ (coinciding with the gap reported in Refs. [22,23]); we observe a fast decay of $\sigma(L)$ with growing $L$, accompanied by $F \rightarrow 1$ (see the blue lines in Figs. 3 and 4 ), indicating the insulating (or semiconducting) behavior. The remaining parameters $\left(\gamma_{3}\right.$ and $\left.\gamma_{4}\right)$ are essentially meaningless in such a case; a slightly elevated conductivity (namely, $\sigma>$ $\left.\sigma_{0}\right)$ is visible for $\gamma_{3}=0.3 \mathrm{eV}$ and $L<100 l_{\perp}$, due to the finite-size effects.

In a gapless case $\left(\delta_{A B}=0\right)$ we identify four apparently different behaviors of $\sigma(L)$, depending on whether the remaining parameters $\left(\gamma_{3}\right.$ and $\left.\gamma_{4}\right)$ take zero- or nonzero values.

For $\delta_{A B}=0$ and $\gamma_{3}=0.3 \mathrm{eV}$, the values of $\sigma(L)$ are generically elevated (above $\sigma_{0}$ ) for any $L$ [black lines in Figs. 3(a) and 3(b)], with the Fano factor $F \approx 1 / 3$ [black lines in Figs. 4(a) and 4(b)]. Starting from $L \approx 2000 l_{\perp}$, one can further distinguish the behaviors for $\gamma_{4}=0.15 \mathrm{eV}$ [solid black line in Fig. 3(a)], for which $\sigma(L)$ grows approximately linearly with $L$, and for $\gamma_{4}=0$ [dashed black line], for which $\sigma(L)$ approaches the value of $3 \sigma_{0}$.

For $\gamma_{4}=0.15 \mathrm{eV}$, the energy shift of three secondary Dirac cones [see Fig. 2(a)] is equal to

$$
\Delta E_{l}=\frac{\hbar^{2} k_{l}^{2} v_{4}}{m v_{0}}=2 \frac{\gamma_{1} \gamma_{3}^{2} \gamma_{4}}{\gamma_{0}^{3}} \approx 0.33 \mathrm{meV}
$$

where $m=\gamma_{1} / 2 v_{0}^{2}$ and $k_{l}=\gamma_{1} v_{3} / \hbar v_{0}^{2}$, leading to a nonzero number of propagating modes (open channels) at zero energy, 

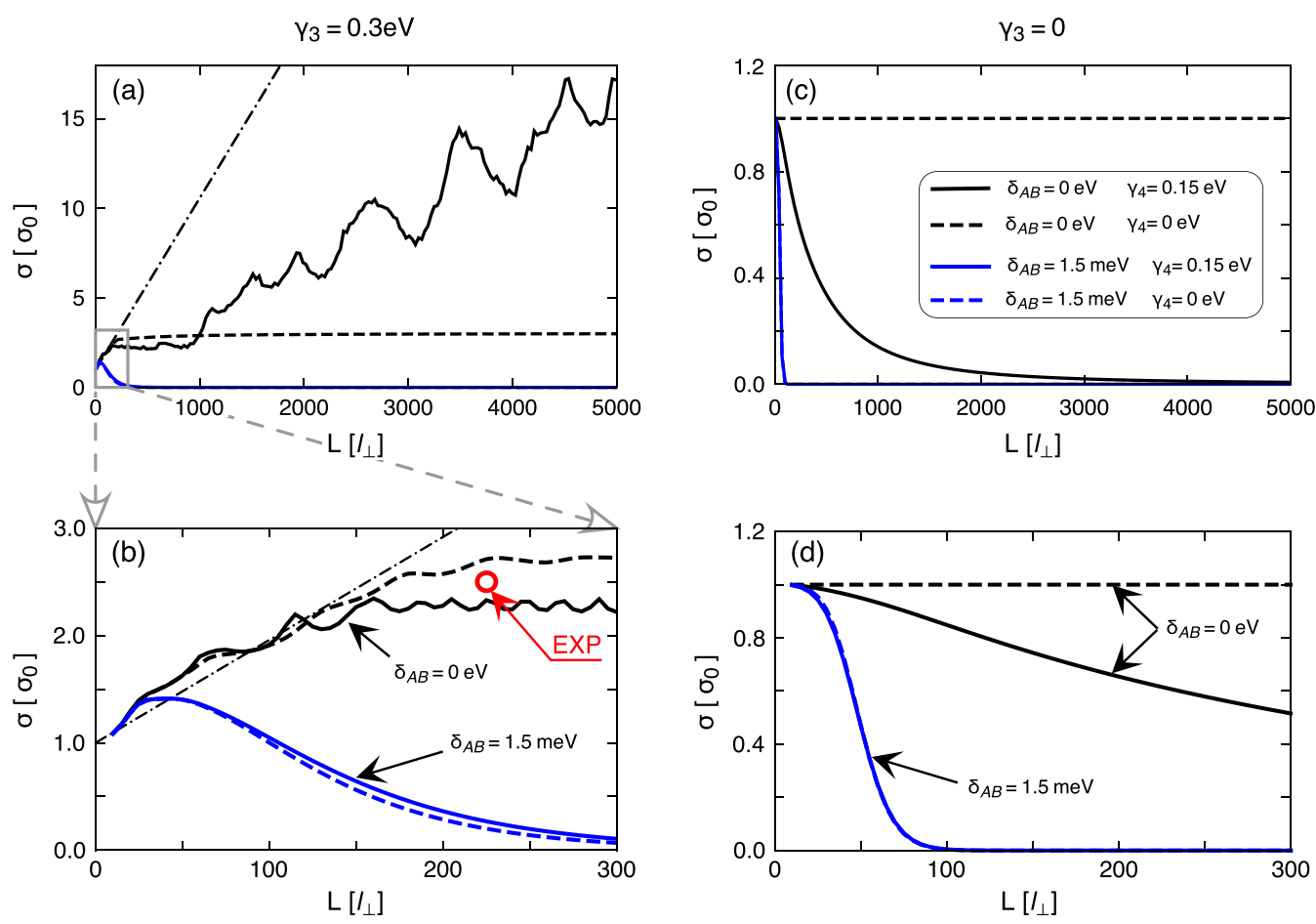

FIG. 3. Conductivity [in units of $\sigma_{0}=8 e^{2} /(\pi h)$ ] as a function of length $L$ (in units of $l_{\perp}=\hbar v_{0} / \gamma_{1}=1.77 \mathrm{~nm}$ ) at a fixed $W / L=20$. The trigonal warping strength is $(\mathrm{a}, \mathrm{b}) \gamma_{3}=0.3 \mathrm{eV}$ or $(\mathrm{c}, \mathrm{d}) \gamma_{3}=0$. Bottom panels are zoom-ins, for small $L$, of the data presented in top panels. Solid (or dashed) lines in all panels are for $\gamma_{4}=0.15 \mathrm{eV}$ (or $\gamma_{4}=0$ ); the staggered potential is $\delta_{A B}=0$ (black lines) or $\delta_{A B}=1.5 \mathrm{meV}$ (blue lines), as indicated with arrows in (b) and (d). The red circle in (b) marks the experimental results of Ref. [29]. Dash-dotted lines in (a) and (b) depict the approximate upper bound given by Eq. (7) in the main text. The line-color encoding shown in (c) is same for all panels.

which can be approximated as [28]

$$
N_{\text {open }}(E=0) \approx 0.68 \frac{\Delta E_{l} W}{\hbar v_{3}}
$$
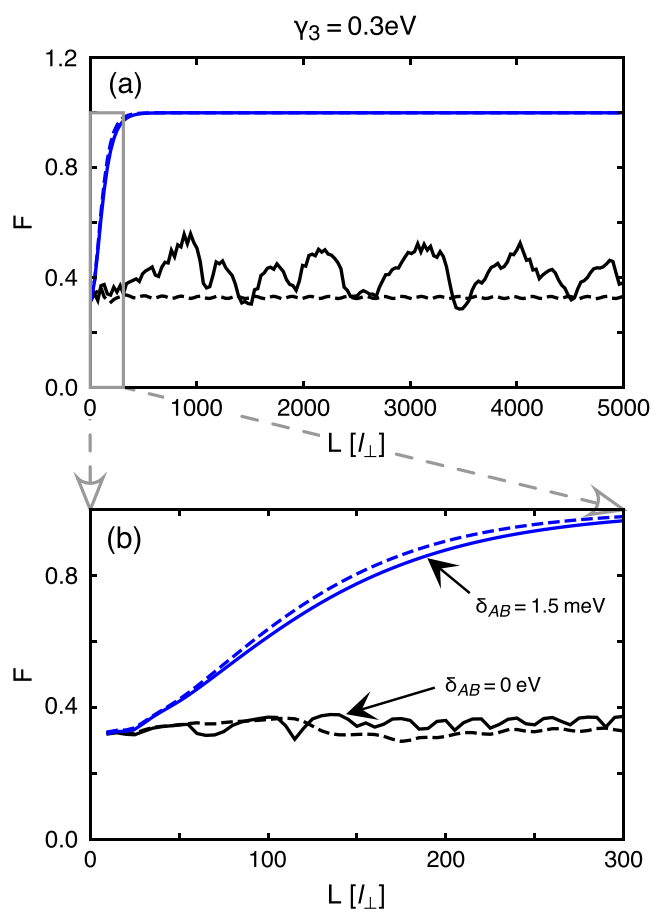

Subsequently, the excess conductivity from secondary Dirac cones can roughly be bounded as

$$
\sigma(L)-\sigma_{0} \lesssim \frac{g_{0} N_{\text {open }} L}{W}=9.6 \times 10^{-3} \sigma_{0} \frac{L}{l_{\perp}},
$$
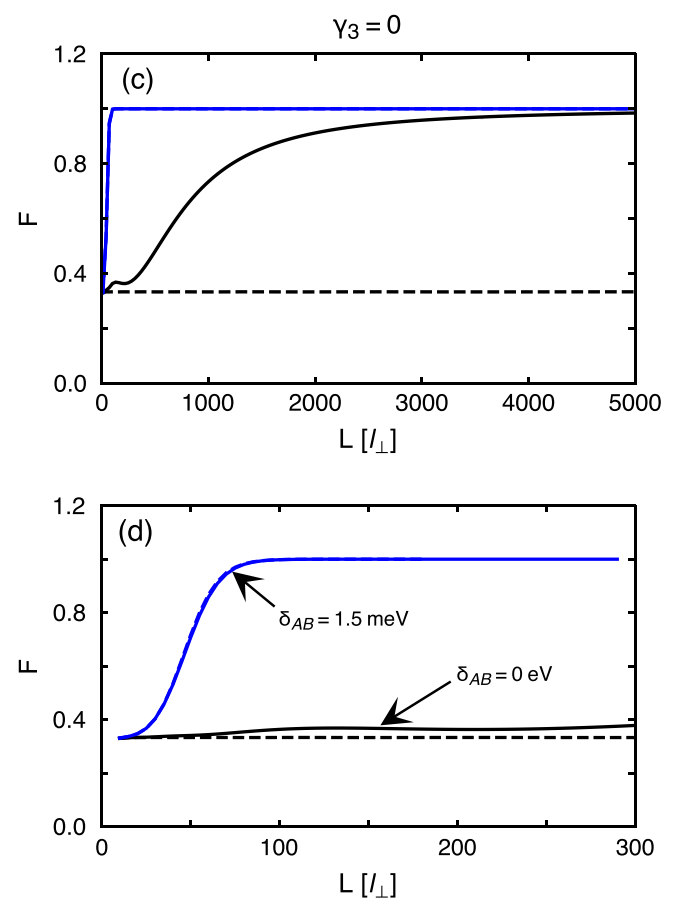

FIG. 4. (a)-(d) The Fano factor $F$ as a function of $L$. The system parameters and the line-color encoding are the same as in Fig. 3. 
with the rightmost equality corresponding to $W / L=20$ and $\sigma_{0}$ on the left-hand side representing a contribution from evanescent waves in the primary Dirac cone [see the dashdotted lines in Figs. 3(a) and 3(b)]. The transmission reduction for propagating modes, approximately by a factor of 2 , can be attributed to the additional backscattering appearing in the double-barrier geometry, which is usually much weaker for a single barrier [31]. A secondary feature of $\sigma(L)$ is a quasiperiodic oscillation due to the Fabry-Pérot resonances appearing for $L=n \Delta L$, where $n=1,2, \ldots$, and (up to the order of magnitude) $\Delta L \sim \pi \hbar v_{3} / \Delta E_{l} \approx 340 l_{\perp}$ [28].

None of these effects is present for $\gamma_{4}=0$, for which the conductivity follows the scenario earlier described in Refs. [12,13]. (In Appendix A, we present the analytical derivation explaining why $\sigma(L) \rightarrow 3 \sigma_{0}$ for $L \rightarrow \infty$ and arbitrarily small $\gamma_{3} \neq 0$.) We further notice that the available experimental value in Ref. [29], reporting $\sigma \approx 2.5 \sigma_{0}$ for $L \approx$ $400 \mathrm{~nm}=226 l_{\perp}$ [red circle in Fig. 3(b)], is equally close to both the results for $\gamma_{4}=0$ and $0.15 \mathrm{eV}$, and the determination of $\gamma_{4}$ via conductivity measurements requires a sample length exceeding $L \gtrsim 2 \mu \mathrm{m}$.

For $\delta_{\mathrm{AB}}=\gamma_{3}=0$, the conductivity behavior with growing $L$ is a bit more peculiar.

If $\gamma_{4}=0$, we simply have $\sigma(L)=\sigma_{0}$ and $F=1 / 3$ for any $L \gg l_{\perp}$ [see dashed black lines in Figs. 3(c), 3(d), 4(c), and 4(d)], reproducing the analytical results of Refs. $[9,10]$.

In contrast, if $\gamma_{4}=0.15 \mathrm{eV}$ (solid black lines) we observe a slow power-law decay of $\sigma(L)$ with growing $L$, which can be approximated as $\sigma(L) \propto L^{-2.0}$ for $L \gtrsim 1000 l_{\perp}$, accompanied by $F \rightarrow 1$. Notice that the Fano factor is $F \approx 1 / 3$ in the range of $L \leqslant 300 l_{\perp}$ shown in Fig. 4(d); the convergence to 1 becomes visible for $L \gtrsim 1000 l_{\perp}$ [see Fig. 4(c)]. Unlike for nonrelativistic electrons [32], we still obtain a finite $\sigma(L)$ in the limit of infinite doping in the leads at fixed $W$ and $L$, signaling the relativistic nature of charge carriers. The vanishing conductivity for $L \rightarrow \infty$ at a fixed $W / L$, in the absence of a gap, clearly represents a remarkable feature of the results, providing an opportunity to verify the $\gamma_{3}=0$ model as put forward in Ref. [21] within ballistic transport experiments. A further reasoning that such a behavior appears generically for $\gamma_{3}=0$ and $\gamma_{4} \neq 0$ is given in Appendix B.

\section{B. Finite-temperature effects}

For $T>0$ and in the linear-response regime the electronic noise is dominated by the Nyquist-Johnson term of $S(0) \approx 4 k_{B} T \sigma W / L$ [27], and the Fano factor becomes irrelevant. Therefore, we limit our discussion to the temperaturedependent conductivity, which is given by

$$
\sigma(T>0)=\frac{g_{0} L}{W} \int d E \operatorname{Tr}\left(\mathbf{t t}^{\dagger}\right)\left(-\frac{\partial f_{\mathrm{FD}}}{\partial E}\right),
$$

where $f_{\mathrm{FD}}(\mu, T, E)=\left[\exp \left((E-\mu) / k_{B} T\right)+1\right]^{-1}$ is the Fermi-Dirac distribution function for a given chemical potential $\mu$, and the remaining symbols are the same as in Eq. (3).

Numerical integration in Eq. (8) is performed, for $\mu=0$, by taking the energy range of $-E_{\mathrm{M}} \leqslant E \leqslant E_{\mathrm{M}}$, with a cutoff energy $E_{\mathrm{M}}=0.05 \mathrm{eV}$ (i.e., $E_{M}>48 k_{B} T$ for $T \leqslant 12 \mathrm{~K}$ ) being sufficiently high to reach a convergence up to the machine roundoff errors. Additionally, when calculating the transmission matrix $\mathbf{t}(E)$, we parametrize the staggered potential in the effective Hamiltonian $H_{\mathrm{BLG}}(2)$ as follows:

$$
\delta_{A B}(T)=\delta_{A B}(0) \begin{cases}\tanh \left(1.74 \sqrt{\frac{T_{C}}{T}-1}\right) & \text { if } T \leqslant T_{C} \\ 0 & \text { if } T>T_{C},\end{cases}
$$

with $T_{C}=12 \mathrm{~K}$ and $\delta_{A B}(0)=1.5 \mathrm{meV}$ reproducing the temperature dependence of a gap reported in Refs. [22,23]. (The gapless case $\delta_{A B}(0)=0$ is considered separately.)

Our numerical results, for $T=0$ and the selected temperatures $0<T \leqslant T_{C}$, are presented in Fig. 5. Similarly as in the previous section, the data sets for $\gamma_{3}=0.3 \mathrm{eV}$ [see Figs. 5(a) and 5(b)] and for $\gamma_{3}=0$ [see Figs. 5(c) and 5(d)] are displayed separately. This time, we limit the presentation to the $\gamma_{4}=0.15 \mathrm{eV}$ case for clarity (solid lines in all panels), as the curves for $\gamma_{4}=0$ closely follow their $\gamma_{4}=0.15 \mathrm{eV}$ counterparts, with the exception of $\gamma_{3}=\delta_{A B}(0)=0$ and $T=$ 0 [see dashed line in Fig. 5(c)], when the conductivity suppression in the presence of EHS symmetry breaking $\left(\gamma_{4} \neq 0\right)$ is clearly visible.

An apparent feature of the results presented in Fig. 5(a) is that the curves for different temperatures closely follow each other up to $L \lesssim 120 l_{\perp}$, above which $\sigma(L)$ grows noticeably faster with $L$ for higher $T$. [Notice that the $T=0$ curve also shows approximately linear growths with $L$, which manifests itself for $L \gtrsim 10^{3} l_{\perp}$; see Fig. 3(a).] The position of a coalescence point, $L \approx 120 l_{\perp}$, can be attributed to fact that above such a length the quantum-size effects are less significant, allowing the finite-temperature effects to dominate transport properties. This can be rationalized taking into account the time-energy uncertainty relation limiting the energy resolution,

$$
\delta E \geqslant \frac{\hbar}{2 \tau_{\text {flight }}}=\frac{\hbar v_{3}}{2 L},
$$

with $\tau_{\text {flight }} \approx L / v_{3}$ being the ballistic time of flight [28], together with the fact that the energy of thermal excitations is $k_{B} T \gtrsim \Delta E_{l}$ for $T \gtrsim 4 \mathrm{~K}$, with $\Delta E_{l}$ given by Eq. (5). Subsequently, one can expect that the propagating modes in secondary Dirac cones are employed (by thermal excitations) provided that $\delta E \ll \Delta E_{l} \lesssim k_{B} T$, leading to

$$
\frac{L}{l_{\perp}} \gg \frac{\gamma_{0}^{2}}{4 \gamma_{3} \gamma_{4}} \approx 55 \text {. }
$$

For $\gamma_{3}=0$ the above reasoning no longer applies; however, a relatively flat $\sigma$ dependence on $L$ for $T>0$ [see Fig. 5(c)] coincides with the divergent lower bound for $L$ in Eq. (11). In such a case, one should rather estimate the time of flight (up to the order of magnitude) as $\tau_{\text {flight }} \sim L / v_{0}$. In turn, the condition $k_{B} T \gg \delta E$ allowing the conductivity enhancement by thermal excitations is equivalent to

$$
\frac{L}{l_{\perp}} \gg \frac{\gamma_{1}}{2 k_{B} T} \approx \frac{2200 \mathrm{~K}}{T},
$$

giving, for instance, $L \gg 440 l_{\perp}$ for $T=5 \mathrm{~K}$. The lower bound for $L$ in Eq. (12) allows one to understand why temperature effects on $\sigma(L)$ are noticeably weakened for $\gamma_{3}=0$, comparing to the $\gamma \neq 0$ case. 

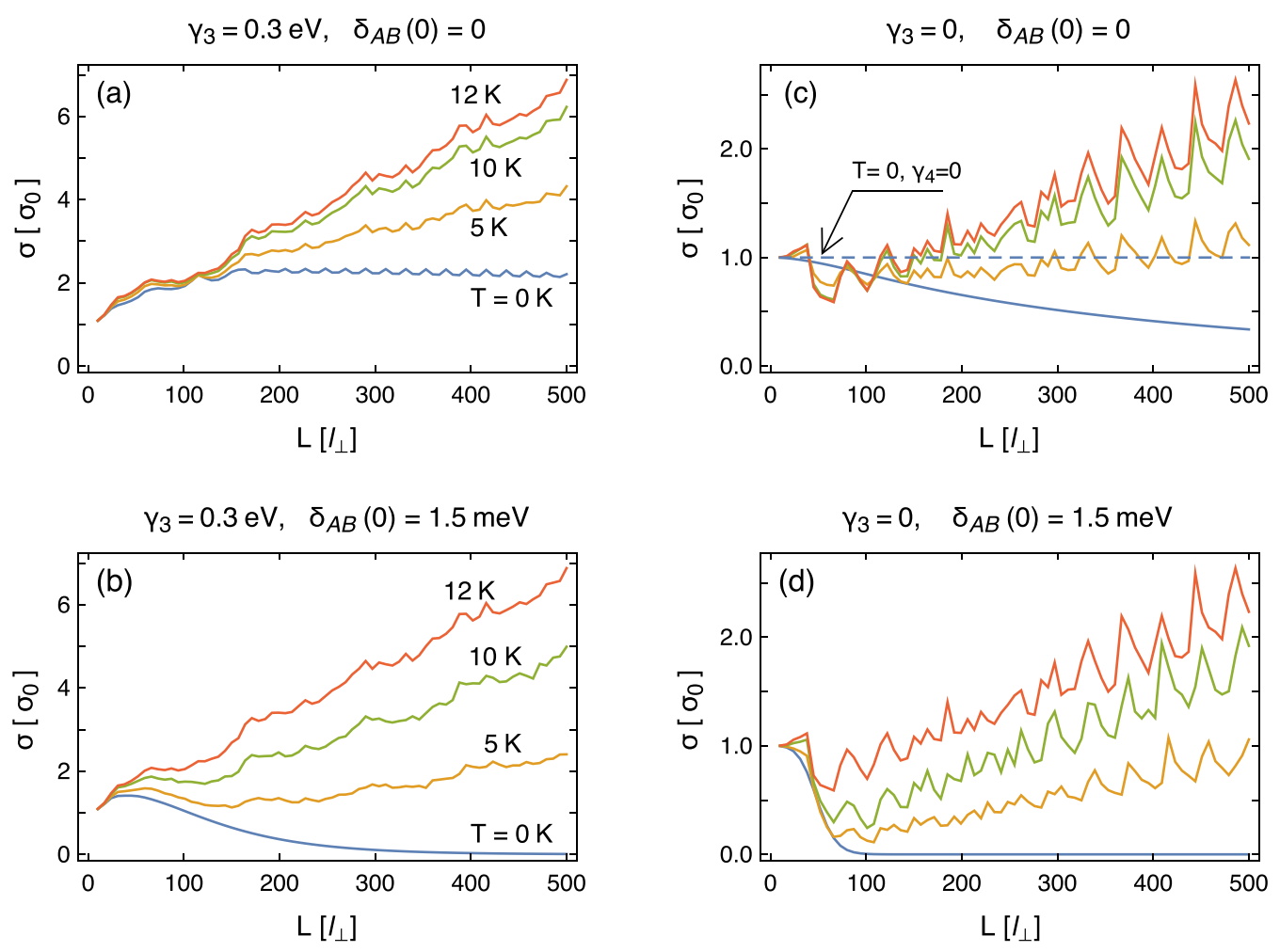

FIG. 5. (a)-(d) Conductivity as a function of $L$ for temperatures $T=0 \mathrm{~K}, T=5 \mathrm{~K}, T=10 \mathrm{~K}$, and $T=T_{C}=12 \mathrm{~K}$ [see Eq. (9) in the main text] indicated by labels in (a) and (b) for all panels. The parameters $\gamma_{3}$ and $\delta_{A B}(0)$ are specified for each panel; the parameter $\gamma_{4}=0.15 \mathrm{eV}$ for all lines, with the exception of a dashed line for $T=0$ in (c), for which $\gamma_{4}=0$ (as marked with an arrow).

In the presence of a staggered potential, $\delta_{A B}(0)=1.5 \mathrm{meV}$, the primary temperature effects on $\sigma(L)$ visible in Figs. 5(b) and 5(d) can be attributed to the gap closing for $T$ approaching $T_{C}$ [see Eq. (9)]. The characteristic system length, above which the value of $\delta_{A B}$ becomes significant, derived from the condition for the energy uncertainty $\delta E \ll \delta_{A B}(0)$, reads

$$
\frac{L}{l_{\perp}} \gg \begin{cases}\frac{1}{2} \gamma_{1} \gamma_{3} / \gamma_{0} \delta_{A B}(0) \approx 12.1 & \text { if } \gamma_{3} \neq 0 \\ \frac{1}{2} \gamma_{1} / \delta_{A B}(0) \approx 127 & \text { if } \gamma_{3}=0,\end{cases}
$$

where we have estimated $\tau_{\text {flight }} \approx L / v_{3}$ (if $\gamma_{3} \neq 0$ ) or $\tau_{\text {flight }} \sim$ $L / v_{0}$ (if $\gamma_{3}=0$ ). This time, our numerical results show that the temperature effects are visible for significantly shorter systems in the $\gamma_{3}=0.3 \mathrm{eV}$ case, comparing to the $\gamma_{3}=0$ case, in a qualitative agreement with the estimation given in Eq. (13).

\section{CONCLUDING REMARKS}

We have investigated, by calculating ballistic transport characteristics within the Landauer-Büttiker formalism, the role of symmetry-breaking terms in the effective Hamiltonian for bilayer graphene. Three such terms, the trigonal warping $\left(\gamma_{3}\right)$, the electron-hole symmetry-breaking interlayer hopping $\left(\gamma_{4}\right)$, and the staggered potential $\left(\delta_{A B}\right)$ quantifying a spontaneous band gap, are independently switched on and off, resulting in different behaviors of the conductivity $(\sigma)$ and the Fano factor $(F)$ with the increasing system length $(L)$ at a fixed width-to-length ratio $(W / L)$.
In the absence of a gap $\left(\delta_{A B}=0\right)$, one can identify three different quantum-transport regimes characterized by the pseudodiffusive shot-noise power, $F=1 / 3$ : (i) the standard pseudodiffusive regime, characterized by $\sigma(L)=\sigma_{0}$ [with $\sigma_{0}=8 e^{2} /(\pi h)$ being a double conductivity of a monolayer] and occurring for $\gamma_{3}=\gamma_{4}=0$, (ii) the asymptotic pseudodiffusive regime, with $\sigma(L) \rightarrow 3 \sigma_{0}$ for $L \rightarrow \infty$, occurring for $\gamma_{3} \neq 0$ and $\gamma_{4}=0$, and (iii) the divergent pseudodiffusive regime, with $\sigma(L) \rightarrow \infty$ for $L \rightarrow \infty$, occurring for $\gamma_{3} \neq 0$ and $\gamma_{4} \neq 0$. Additionally, for $\gamma_{3}=0$ and $\gamma_{4} \neq 0$, the system can be regarded as a marginal conductor, with $\sigma(L) \rightarrow 0$ (showing a power-law decay) and $F \rightarrow 1$ for $L \rightarrow \infty$.

In the presence of a staggered potential at $T=0\left(\delta_{A B}(0)>\right.$ 0 ), a semiconducting behavior is observed regardless of the remaining parameters $\left(\gamma_{3}\right.$ and $\gamma_{4}$ ); i.e., $\sigma(L) \rightarrow 0$ (showing the exponential decay) and $F \rightarrow 1$ for $L \rightarrow \infty$. For $T>0$, a zero-gap behavior is gradually restored (for any combination of $\gamma_{3}$ and $\gamma_{4}$ ) when the energy of thermal excitations $k_{B} T \gtrsim$ $\delta_{A B}(0)$.

We hope that our numerical results will help verify the bilayer graphene models proposed in the literature, as soon as ballistic samples of the length $L \gtrsim 1 \mu \mathrm{m}$ become available. So far, conductivity measurements for shorter samples [29] suggest that the models neglecting the trigonal warping $\left(\gamma_{3}=0\right)$ cannot correctly reproduce transport properties in the mesoscopic range, but conclusive information concerning the value of $\gamma_{4}$ is missing.

Apart from the material-science aspects outlined above, the asymptotic conductivity behavior suggests that bilayer graphene represents a model case when discussing the 
generality of spontaneous symmetry breaking in quantum systems [33,34]. When $\sigma$ is considered as an order parameter, our findings can be summarized by putting forward the noncommuting order of limits, as $L \rightarrow \infty$ and the relevant symmetry breakings vanish, namely,

$$
\begin{gathered}
\lim _{L \rightarrow \infty} \lim _{d \rightarrow \infty} \lim _{\delta_{A B} \rightarrow 0} \sigma=\lim _{L \rightarrow \infty} \lim _{\delta_{A B} \rightarrow 0} \lim _{d \rightarrow \infty} \sigma=\sigma_{0}, \\
\lim _{d \rightarrow \infty} \lim _{L \rightarrow \infty} \lim _{\delta_{A B} \rightarrow 0} \sigma=\infty, \\
\lim _{d \rightarrow \infty} \lim _{\delta_{A B} \rightarrow 0} \lim _{L \rightarrow \infty} \sigma=\lim _{\delta_{A B} \rightarrow 0}[\cdots] \sigma=0,
\end{gathered}
$$

where we have introduced the distance between the layers $d$ (with $d \rightarrow \infty$ corresponding to simultaneous limits of $\gamma_{3} \rightarrow 0$ and $\gamma_{4} \rightarrow 0$ [35]), and the dots [‥ in Eq. (16) mark that the order of the two remaining limits is arbitrary in such a case. From this perspective, it becomes clear that both the sublattice and the combined rotationalelectron-hole symmetry breakings may appear spontaneously, as consequences of the layer stacking in graphene $(d=$ const $<\infty)$.

The peculiar cases of $\gamma_{3} \neq 0$ or $\gamma_{4} \neq 0$ in the absence of other symmetry breakings (i.e., $\delta_{A B}=\gamma_{4}=0$ or $\delta_{A B}=\gamma_{3}=$ 0 ) do not seem to have as clear a physical interpretation. However, in heterostructures containing graphene, a variety of spontaneous symmetry breakings may appear due to the couplings to surrounding layers, encouraging one to consider also anomalous parameter configurations.

Our considerations are limited to Bernal-stacked bilayer graphene, since other stacking usually opens the band gap leading to the vanishing conductivity. One notable exception is the magic-angle twisted bilayer graphene [36-40] showing a gapless band structure. However, the role of electron-electron interaction in such a system is more significant; in particular, the ground state near the chargeneutrality point is a correlated insulator [38-40]. Therefore, we expect the vanishing zero-energy conductivity to appear generically in bilayer graphene for non-Bernal stackings.

\section{ACKNOWLEDGMENTS}

The work was supported by the National Science Centre of Poland (NCN) via Grant No. 2014/14/E/ST3/00256. Computations were partly performed using the PL-Grid infrastructure.

\section{APPENDIX A: TRANSMISSION THROUGH BILAYER GRAPHENE IN THE PRESENCE OF TRIGONAL WARPING $\left(\gamma_{3} \neq 0, \gamma_{4}=0\right)$}

Here, we present the analytical derivation of the total transmission (i.e., transmission probability summed over normal modes), coinciding with the Landauer-Büttiker conductivity [see Eq. (3) in the main text] $\sigma(L) \rightarrow 3 \sigma_{0}$ in the limit of $L, W \rightarrow \infty$, at $W / L=$ const $\gg 1$. Some partial results were earlier reported in Ref. [12], but the full derivation, to our best knowledge, is missing in the literature.
The dispersion relation for the Hamiltonian given by Eq. (2) in the main text, with $\delta_{A B}=\gamma_{4}=0$, takes the form

$$
\begin{aligned}
E^{2} & =\frac{\gamma_{1}^{2}}{2}+\left(v_{0}^{2}+\frac{v_{3}^{2}}{2}\right) p^{2} \pm \sqrt{\Gamma}, \\
\Gamma= & \frac{1}{4}\left(\gamma_{1}^{2}-v_{3}^{2} p^{2}\right)^{2}+v_{0}^{2} p^{2}\left(\gamma_{1}^{2}+v_{3}^{2} p^{2}\right) \\
& +2 \gamma_{1} v_{3} v_{0}^{2} p^{3} \cos (3 \varphi),
\end{aligned}
$$

where $p=\sqrt{p_{x}^{2}+p_{y}^{2}}$ and we have set $\theta=0$ for simplicity (later, we show that the physical results are independent of the lattice orientation in the $L \rightarrow \infty$ limit).

In the vicinity of zero energy $(|E| \rightarrow 0)$, there are four solutions of the above equation corresponding to four Dirac cones: the central cone, located at $\mathbf{p}=\left(p_{x}, p_{y}\right)=(0,0)$, and three satellite cones, located (in polar coordinates) at $p=$ $\gamma_{1} v_{3} / v_{0}^{2}, \varphi=0,2 \pi / 3,4 \pi / 3$. Below, we calculate the transmission of the system assuming that the states corresponding to different Dirac cones do not interfere among themselves. Physically, such a supposition corresponds to the conditions for the energy and system sizes

$$
|E|, \frac{\hbar v_{3}}{L}, \frac{\hbar v_{3}}{W} \ll E_{L},
$$

where the Lifshitz energy $E_{L}=\frac{1}{4} \gamma_{1}\left(v_{3} / v_{0}\right)^{2}$. For $\gamma_{0}=$ $3.16 \mathrm{eV}, \gamma_{1}=0.381 \mathrm{eV}$, and $\gamma_{3}=0.3 \mathrm{eV}$ [24], we have $E_{L} \approx$ $1 \mathrm{meV}$, and the last two conditions in Eq. (A3) are equivalent to $L, W \gg 41_{\perp} \gamma_{3} / \gamma_{0}=75 \mathrm{~nm}$.

Expanding the dispersion relation given by Eqs. (A3) and (A4) up to second order around $\mathbf{p}=(0,0)$, we obtain

$$
E^{2}=v_{3}^{2}\left(p_{x}^{2}+p_{y}^{2}\right) \text {. }
$$

Thus, the central Dirac cone has an isotropic dispersion relation, closely resembling the dispersion relation following from the monolayer graphene Hamiltonian [see Eq. (1) in the main text]; in fact, the only difference is the proportionality coefficient $v_{3}$ instead of $v_{F}$.

Now, we write the effective single-cone Hamiltonian, corresponding to the dispersion relation given by Eq. (A4):

$$
H_{\text {central }}=\left(\begin{array}{cc}
0 & v_{3} \pi \\
v_{3} \pi^{\dagger} & 0
\end{array}\right) \text {. }
$$

Solving the scattering problem for a rectangular sample described by the above Hamiltonian with heavily (infinitely) doped leads one gets the formula for the transmission coefficient as a function of the transverse momentum $\left(k_{y}=p_{y} / \hbar\right)$ :

$$
T\left(k_{y}\right)=\frac{1}{\cosh ^{2}\left(k_{y} L\right)} .
$$

For the periodic boundary conditions, the transverse momentum gets quantized values, $k_{y}^{(j)}=2 \pi j / W$, with $j=$ $0, \pm 1, \pm 2, \ldots$. For $W \gg L$, one can approximate the sum over $k_{y}^{(j)}$ by an integral, obtaining the total transmission

$$
\sum_{j} T\left(k_{y}^{(j)}\right) \approx \frac{W}{2 \pi} \int_{-\infty}^{+\infty} T\left(k_{y}\right) d k_{y}=\frac{1}{\pi} \frac{W}{L} .
$$

Next, we expand (up to second order) the dispersion relation around $\mathbf{p}=\left(\gamma_{1} v_{3} / v_{0}^{2}, 0\right)$ (i.e., the satellite Dirac cone at 
$\varphi=0)$, arriving at

$$
E^{2}=\frac{v_{3}^{2}}{\left(1+\left(v_{3} / v_{0}\right)^{2}\right)^{2}}\left(p_{x}^{2}+9 p_{y}^{2}\right) .
$$

The corresponding single-cone Hamiltonian reads

$$
H_{\text {satellite }}^{(\varphi=0)}=\left(\begin{array}{cc}
0 & v_{3}\left(p_{x}+i 3 p_{y}\right) \\
v_{3}\left(p_{x}-i 3 p_{y}\right) & 0
\end{array}\right) .
$$

This time, solving the scattering problem for a rectangular sample we get the transmission coefficient

$$
T\left(k_{y}\right)=\frac{1}{\cosh ^{2}\left(3 k_{y} L\right)},
$$

and the integration over $k_{y}$ leads to

$$
\frac{W}{2 \pi} \int_{-\infty}^{+\infty} T\left(k_{y}\right) d k=\frac{1}{3 \pi} \frac{W}{L} .
$$

The calculations for remaining Dirac cones at $\varphi \neq 0$ are more involved, yet straightforward. Generalizing the above reasoning for $\mathbf{p}=\gamma_{1}\left(v_{3} / v_{0}^{2}\right)(\cos \varphi, \sin \varphi)$, we get

$$
E^{2}=\frac{v_{3}^{2}}{\left(1+\left(v_{3} / v_{0}\right)^{2}\right)^{2}}\left[p_{x}^{2}+p_{y}^{2}+8\left(p_{x} \sin \varphi+p_{y} \cos \varphi\right)^{2}\right]
$$

and

$$
H_{\text {satellite }}^{(\varphi)}=\left(\alpha \sigma_{x}+\beta \sigma_{y}\right)
$$

where

$$
\begin{gathered}
\alpha=p_{x} \cos \varphi-p_{y} \sin \varphi, \\
\beta=-3 p_{x} \sin \varphi-3 p_{y} \cos \varphi .
\end{gathered}
$$

Finally, we have

$$
T\left(k_{y}\right)=\frac{1}{\cosh ^{2}\left[3 k_{y} L /(5-4 \cos (2 \varphi))\right]}
$$

and

$$
\frac{W}{2 \pi} \int_{-\infty}^{+\infty} T\left(k_{y}\right) d k=\frac{5-4 \cos (2 \varphi)}{3 \pi} \frac{W}{L} .
$$

Summing up the contributions from all four Dirac cones, we obtain the total transmission

$$
T_{\text {total }}=\frac{6 W}{\pi L} .
$$

Substituting the above into Eq. (3) in the main text, we obtain $\sigma=3 \sigma_{0}$ in physical units. Remarkably, the result is independent of the lattice orientation, as we have $\cos (\varphi)+$ $\cos (\varphi+4 \pi / 3)+\cos (\varphi-4 \pi / 3)=0$ for any real value of $\varphi$. (Notice that the summation of independent contributions from four Dirac cones, performed above, instantly reproduces the limit of $L, W \rightarrow \infty$.)

Similarly, for the Fano factor we have

$$
F=1-\frac{\sum_{\text {cones }} \int d k_{y}\left[T\left(k_{y}\right)\right]^{2}}{\sum_{\text {cones }} \int d k_{y} T\left(k_{y}\right)}=\frac{1}{3} .
$$
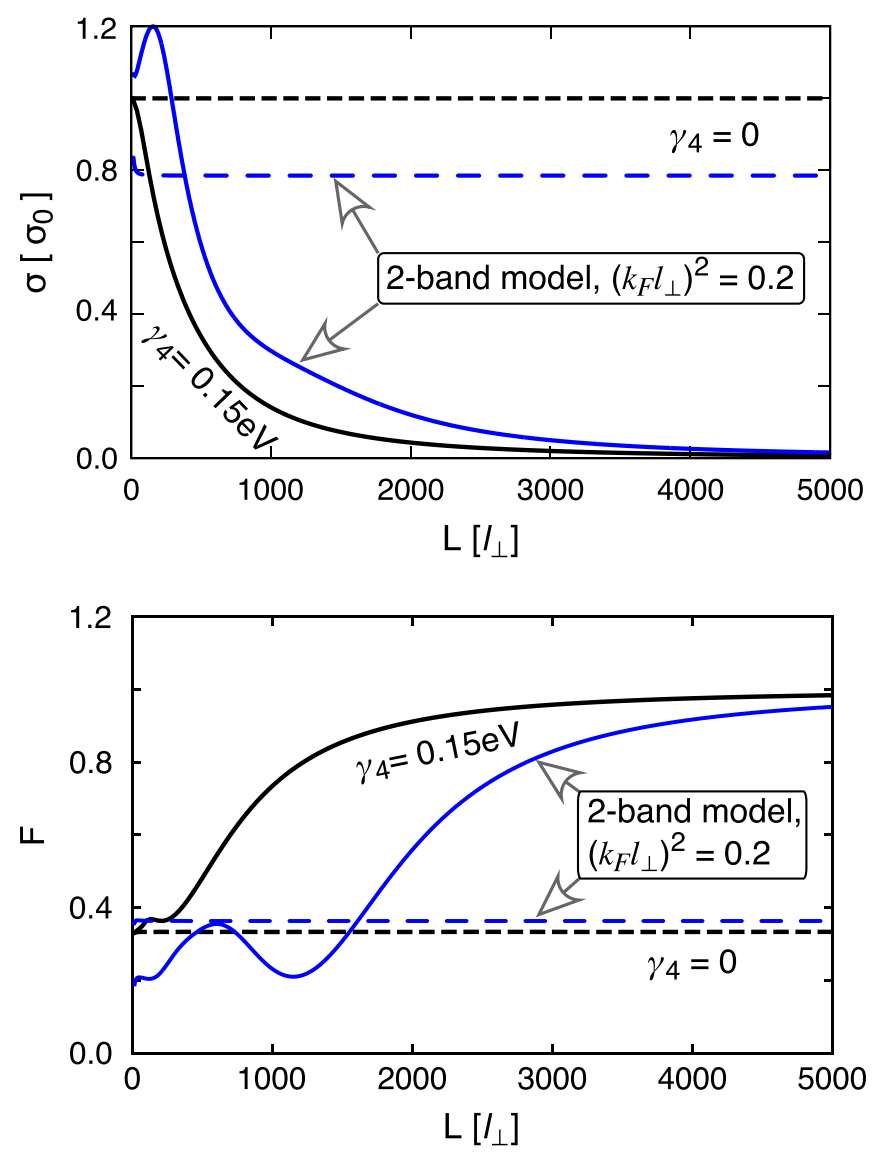

FIG. 6. Conductivity (top) and the Fano factor (bottom) obtained from Eq. (B3) for $\left(k_{F} l_{\perp}\right)^{2}=0.2$ and $\gamma_{4}=0.15 \mathrm{eV}$ (solid blue lines), or $\gamma_{4}=0$ (dashed blue lines). The corresponding results for the fourband model are reproduced from Figs. 3(c) and 4(c) for a comparison (solid and dashed black lines).

\section{APPENDIX B: THE EFFECT OF $\gamma_{4} \neq 0$ TUNNELING IN \\ THE ABSENCE OF TRIGONAL WARPING $\left(\gamma_{3}=0\right)$}

In this section, we consider the case complementary to the analyzed in Appendix A. The Hamiltonian given by Eq. (2) in the main text, for $\delta_{A B}=\gamma_{3}=0$, reduces to

$$
H_{\mathrm{BLG}}=\left(\begin{array}{cccc}
0 & v_{0} \pi & \gamma_{1} & -v_{4} \pi^{\dagger} \\
v_{0} \pi^{\dagger} & 0 & -v_{4} \pi^{\dagger} & 0 \\
\gamma_{1} & -v_{4} \pi & 0 & v_{0} \pi^{\dagger} \\
-v_{4} \pi & 0 & v_{0} \pi & 0
\end{array}\right),
$$

leading to the two low-energy bands (with $E=0$ for $p=0$ ) and the two high-energy bands (with $E= \pm \gamma_{1}$ for $p=0$ ) in the dispersion relation. For low energies, one can write the effective two-band Hamiltonian (see Ref. [16])

$$
H_{2 \text { band }}=\frac{1}{2 m}\left(\begin{array}{cc}
\mu_{4} \pi \pi^{\dagger} & -(\pi)^{2} \\
-\left(\pi^{\dagger}\right)^{2} & \mu_{4} \pi^{\dagger} \pi
\end{array}\right),
$$

where $m=\gamma_{1} / 2 v_{0}^{2}$ and $\mu_{4}=4 m v_{0} v_{4} / \gamma_{1}=2 v_{4} / v_{0}$.

Now, we follow the approach proposed by Katsnelson in Ref. [9], performing the mode matching for two interfaces between heavily and weakly doped areas (the leads and the sample), separated by a distance $L$. For a fixed but finite 
doping in the leads (quantified by the Fermi wavenumber $k_{F}$ ), elementary analysis leads to the following formula for the transmission coefficient for a given transverse wavenumber $k_{y}$ (conserved at both the interfaces):

$$
T\left(k_{y}\right)=\frac{16 \zeta^{2}\left\{\left(-1-\mu_{4}\right) k_{F} L \zeta \cosh (\zeta)-\mu_{4}\left[\left(-1-\mu_{4}\right) k_{F} L+2 \zeta^{2}\right] \sinh (\zeta)\right\}^{2}}{4 \zeta^{2}\left[\zeta \cosh (2 \zeta)-\mu_{4} k_{F} L \sinh (2 \zeta)\right]^{2}+\left(k_{F} L\right)^{2}\left\{-2 k_{F} L \zeta^{2}+\mu_{4}^{2} k_{F} L[\cosh (2 \zeta)-1]-2 \mu_{4} \zeta \sinh (2 \zeta)\right\}^{2}},
$$

where we have defined $\zeta=k_{y} L$.

Changing the variables according to $T\left(k_{y}\right) \equiv T(\zeta, L)$ we find that the Landauer-Büttiker conductivity, for a fixed $W / L \gg 1$, is bounded by

$$
\sigma(L)=\frac{1}{2 \pi} \int d \zeta T(\zeta, L) \lesssim \frac{\text { const }}{L^{2}} \quad\left(\text { for } \mu_{4} \neq 0\right),
$$

vanishing in the $L \rightarrow \infty$ limit. For $\mu_{4}=0$, the conductivity $\sigma(L) \approx(\pi / 4) \sigma_{0}$, and the Fano factor $F \approx 1-2 / \pi$ for $L \gg$ $l_{\perp}$, being numerically close to the results by Snyman and Beenakker [10].

The approximate upper bound for $\mu_{4} \neq 0$, given in Eq. (B4), is further supported with the numerical results presented in Fig. 6, where we have set the doping in the leads such that $\left(k_{F} l_{\perp}\right)^{2}=0.2$ (after Ref. [10]), and $W / L=20$. Numerical calculations for the full four-band model given the Hamiltonian $H_{\mathrm{BLG}}$ (B1) leads to a noticeably faster but also a power-law decay of the conductivity, which can be approximated as $\sigma(L) \propto L^{-2.0}$ for $L \gtrsim 1000 l_{\perp}$.
[1] M. I. Katsnelson, Eur. Phys. J. B 51, 157 (2006).

[2] J. Tworzydło, B. Trauzettel, M. Titov, A. Rycerz, and C. W. J. Beenakker, Phys. Rev. Lett. 96, 246802 (2006).

[3] F. Miao, S. Wijeratne, Y. Zhang, U. C. Coscun, W. Bao, and C. N. Lau, Science 317, 1530 (2007).

[4] L. DiCarlo, J. R. Williams, Y. Zhang, D. T. McClure, and C. M. Marcus, Phys. Rev. Lett. 100, 156801 (2008).

[5] R. Danneau, F. Wu, M. F. Craciun, S. Russo, M. Y. Tomi, J. Salmilehto, A. F. Morpurgo, and P. J. Hakonen, Phys. Rev. Lett. 100, 196802 (2008).

[6] M. I. Katsnelson, Graphene: Carbon in Two Dimensions (Cambridge University Press, Cambridge, UK, 2012), Chap. 3.

[7] C. W. J. Beenakker, Rev. Mod. Phys. 80, 1337 (2008).

[8] J. Wurm, A. Rycerz, I. Adagideli, M. Wimmer, K. Richter, and H. U. Baranger, Phys. Rev. Lett. 102, 056806 (2009).

[9] M. I. Katsnelson, Eur. Phys. J. B 52, 151 (2006).

[10] I. Snyman and C. W. J. Beenakker, Phys. Rev. B 75, 045322 (2007).

[11] J. Cserti, Phys. Rev. B 75, 033405 (2007).

[12] A. G. Moghaddam and M. Zareyan, Phys. Rev. B 79, 073401 (2009).

[13] G. Rut and A. Rycerz, Europhys. Lett. 107, 47005 (2014).

[14] J. W. McClure, Phys. Rev. 108, 612 (1957).

[15] J. C. Slonczewski and P. R. Weiss, Phys. Rev. 109, 272 (1958).

[16] E. McCann and M. Koshino, Rep. Prog. Phys. 76, 056503 (2013).

[17] S. Das Sarma, Sh. Adam, E. H. Hwang, and E. Rossi, Rev. Mod. Phys. 83, 407 (2011).

[18] The next-nearest layer parameter $\gamma_{2}$ is not present in bilayer graphene (see Refs. $[14,15]$ ).

[19] The limit of $\sigma(L) \rightarrow 3 \sigma_{0}$ for $L \rightarrow \infty$ appears for a generic crystallographic orientation of a sample. In a particular case when the current is passed precisely along a zigzag direction, the limit of $(7 / 3) \sigma_{0}$ is predicted (see Ref. [12]).

[20] W. Bao, J. Velasco, F. Zhang, L. Jing, B. Standley, D. Smirnov, M. Bockrath, A. H. MacDonald, and C. N. Lau, Proc. Natl. Acad. Sci. USA 109, 10802 (2012).
[21] M. Yankowitz, J. I. Wang, S. Li, A. G. Birdwell, Y.A. Chen, K. Watanabe, T. Taniguchi, S. Y. Quek, P. Jarillo-Herrero, and B. J. LeRoy, APL Mater. 2, 092503 (2014).

[22] A. L. Grushina, D.-K. Ki, M. Koshino, A. A. L. Nicolet, C. Faugeras, E. McCann, M. Potemski, and A. F. Morpurgo, Nat. Commun. 6, 6419 (2015).

[23] Y. Nam, D.-K. Ki, M. Koshino, E. McCann, and A. F. Morpurgo, 2D Mater. 3, 045014 (2016).

[24] The values of $\gamma_{0}$ and $\gamma_{1}$ are taken from A. B. Kuzmenko, I. Crassee, D. van der Marel, P. Blake, and K. S. Novoselov, Phys. Rev. B 80, 165406 (2009).

[25] Unlike in monolayer graphene, when the vanishing density of states preserves the semimetallic phase, the Mott-insulating phase may generically appear in the BLG ground state for weak interactions; see J. E. Hirsch, Phys. Rev. B 31, 4403 (1985); L. M. Martelo, M. Dzierzawa, L. Siffert, and D. Baeriswyl, Z. Phys. B 103, 335 (1997).

[26] For the theoretical discussion of a BLG ground-state phase diagram in the presence of interactions, see R. E. Throckmorton and S. Das Sarma, Phys. Rev. B 90, 205407 (2014); J. N. Leaw, H.-K. Tang, P. Sengupta, F. F. Assaad, I. F. Herbut, and S. Adam, ibid. 100, 125116 (2019).

[27] Yu. V. Nazarov and Ya. M. Blanter, Quantum Transport: Introduction to Nanoscience (Cambridge University Press, Cambridge, UK, 2009), Chap. 1.

[28] D. Suszalski, G. Rut, and A. Rycerz, Phys. Rev. B 97, 125403 (2018).

[29] A. S. Mayorov, D. C. Elias, M. Mucha-Kruczyński, R. V. Gorbachev, T. Tudorovskiy, A. Zhukov, S. V. Morozov, M. I. Katsnelson, V. I. Falko, A. K. Geim, and K. S. Novoselov, Science 333, 860 (2011).

[30] R. Kraft, J. Mohrmann, R. Du, P. B. Selvasundaram, M. Irfan, U. N. Kanilmaz, F. Wu, D. Beckmann, H. von Löhneysen, R. Krupke, A. Akhmerov, I. Gornyi, and R. Danneau, Nat. Commun. 9, 1722 (2018).

[31] D. Suszalski, G. Rut, and A. Rycerz, J. Phys.: Condens. Matter 31, 415501 (2019). 
[32] For Schrödinger electrons, incident on a rectangular barrier at zero energy (with respect to the top of the barrier), the transmission probability reads $T_{n}=\left[\cosh ^{2} L q_{n}+\right.$ $\left.\left(k_{\infty}^{2} / 2 q_{n}^{2}-1\right)\left(k_{\infty}^{2} / q_{n}^{2}-1\right)^{-1} \sinh ^{2} L q_{n}\right]^{-1}$, with $q_{n}=2 \pi n / W$ $(n=0,1,2, \ldots)$ being the quantized transverse wavenumber for the periodic boundary conditions, and $k_{\infty}$ denoting the wavenumber in heavily doped leads. For $k_{\infty} \rightarrow \infty$, we have $T_{n} \propto\left(q_{n} / k_{\infty}\right)^{2}$, vanishing for any $n$.

[33] J. van Wezel and J. van den Brink, Am. J. Phys. 75, 635 (2007).

[34] J. van Wezel, J. Zaanen, and J. van den Brink, Phys. Rev. B 74, 094430 (2006).

[35] The effect of increasing distance between the layers $d$ on the tight-binding parameters depicted in Fig. 1(a) can roughly be estimated as $\gamma_{j} \propto \exp \left(-\beta d_{j} / d_{j}^{(0)}\right)$, where the electron-phonon coupling $\beta \approx 2-3$, and $d_{j}\left(d_{j}^{(0)}\right)$ is the actual (equilibrium) distance between the atoms connected by $\gamma_{j}$; see, e.g., M. A. H. Vozmediano, M. I. Katsnelson, and F. Guinea, Phys. Rep. 496, 109 (2010).

[36] R. Bistritzer and A. H. MacDonald, Proc. Natl. Acad. Sci. USA 108, 12233 (2011).

[37] Y. Cao, V. Fatemi, S. Fang, K. Watanabe, T. Taniguchi, E. Kaxiras, and P. Jarillo-Herrero, Nature (London) 556, 43 (2018).

[38] Y. Cao, V. Fatemi, A. Demir, S. Fang, S. L. Tomarken, J. Y. Luo, J. D. Sanchez-Yamagishi, K. Watanabe, T. Taniguchi, E. Kaxiras, Ray C. Ashoori, and P. Jarillo-Herrero, Nature (London) 556, 80 (2018).

[39] C. Xu and L. Balents, Phys. Rev. Lett. 121, 087001 (2018).

[40] M. Fidrysiak, M. Zegrodnik, and J. Spałek, Phys. Rev. B 98, 085436 (2018). 